\title{
Silla turca vacía primaria reversible. A propósito de un caso
}

\author{
J. González-Tortosa; C. Piqueras-Pérez; A. Ruiz-Espejo y J.F. Martínez-Lage
}

Servicio de Neurocirugía. Hospital Universitario Virgen de la Arrixaca. Murcia.

\section{Resumen}

Se presenta el caso de un niña de 13 años con un aumento del tamaño de la hipófisis sugerente de adenoma que, tras un episodio de hidrocefalia aguda, desarrolló en la RM una silla turca vacía parcial. Tras la colocación de una derivación de líquido cefalorraquídeo, se normalizó la presión intracraneal y la hipófisis volvió a ocupar todo el espacio de la fosa sellar. Se discute la posibilidad de que en la silla turca vacía primaria con hipertensión intracraneal crónica idiopática, esté implicada una atrofia isquémica de la adenohipófisis que impide la recuperación del volumen glandular tras la restauración de la PIC a los niveles normales. La reversión de la silla turca vacía es un indicador de la normalización de la PIC.

PALABRAS CLAVE: Silla turca vacía. Hipertensión intracraneal. Hidrocefalia. Derivación de líquido cefalorraquídeo.

\section{Reversible primary empty sella. Case report}

\section{Summary}

We report the case of a 13-year-old girl with an increased hypophysis that mimicked an adenoma, who developed a partial empty sella on MRI after an acute episode of hydrocephalus. After replacement of a CSF shunt, the intracranial pressure returned to normal and the hypophysis filled up again all the sellar fossa. We discuss the possibility of the involvement by an ischemic atrophy of the adenohypophysis in the development of a primary empty sella with idiopathic chronic raised intracranial pressure that prevents the recovery of the gland volume after restoring the intracranial pressure to normal values. Restitution of empty sella may be an indicator of normal intracranial pressure in these cases.

Recibido: Recibido: 10-07-09. Aceptado: 16-11-09
KEY WORDS: Empty sella. Intracranial hypertension. Hydrocephalus. Ventriculoperitoneal shunt.

\section{Introducción}

La silla turca vacía primaria (STVP) es una entidad habitualmente estable en su evolución ${ }^{6}$ y es conocida su frecuente asociación con la hipertensión intracraneal crónica idiopática (HICI) $)^{4,10,17,19,20}$. La razón por la que la glándula hipofisaria permanece disminuida de tamaño una vez que la hipertensión intracraneal se soluciona, es desconocida. Nuestra hipótesis es que la hipertensión intracraneal crónica termina produciendo, con el tiempo, una atrofia glandular que le impide recuperar su tamaño normal y el caso que publicamos apunta a que la elasticidad de la glándula conserva capacidad de recuperación de su volumen en las fases iniciales, cuando aún no se ha establecido la atrofia de su parénquima.

\section{Caso clínico}

Niña de 13 años, operada al nacer por hidrocefalia posthemorrágica asociada a prematuridad mediante una válvula de Hakim. Así mismo está diagnosticada de neurofibromatosis tipo I, presentando neurofibromas cutáneos, manchas de café con leche y antecedentes familiares de la enfermedad en madre y abuelo materno. A los 7 años presentó síntomas de pubertad precoz, por lo que fue sometida a tratamiento con $3,75 \mathrm{mg}$ de triptorelina, una ampolla mensual, hasta la edad de 12 años, presentando la menarquia pocos meses más tarde y continuando con menstruaciones normales hasta ahora. El desarrollo estato-ponderal ha sido normal para su edad.

En una RM realizada para control de su hidrocefalia, se detectó un aumento de la glándula hipofisaria, sugerente de adenoma (Fig 1 A y B).

La agudeza y campos visuales, así como el resto de la exploración neurológica eran normales. Los estudios hor-

Abreviaturas. HICI: hipertensión intracraneal crónica idiopática. STVP: silla turca vacía primaria. 


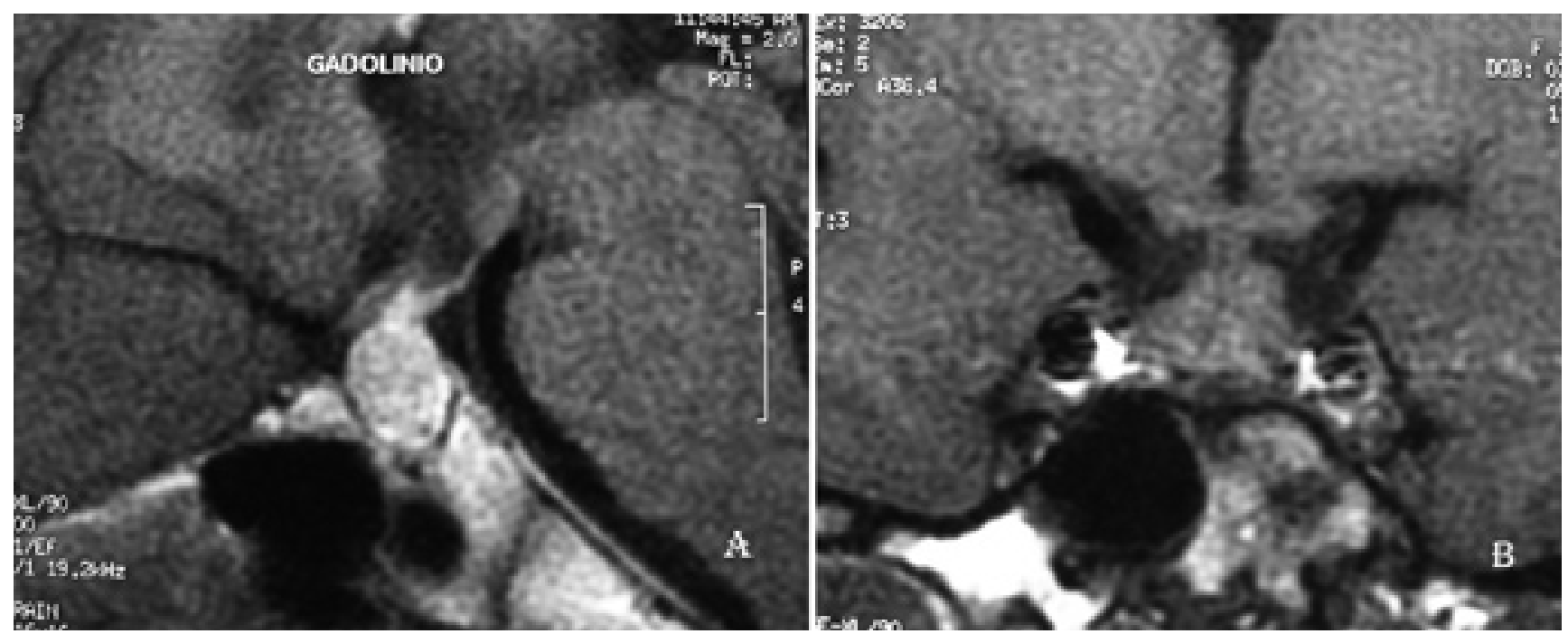

Figura 1. A: RM sagital con contraste: glándula hipofisaria aumentada de tamaño; invade la cisterna supraselar y presenta una captación uniforme de contraste. B: RM T1 coronal: aumento irregular del volumen de la hipófisis, sin desplazamiento lateral del tallo hipofisario.

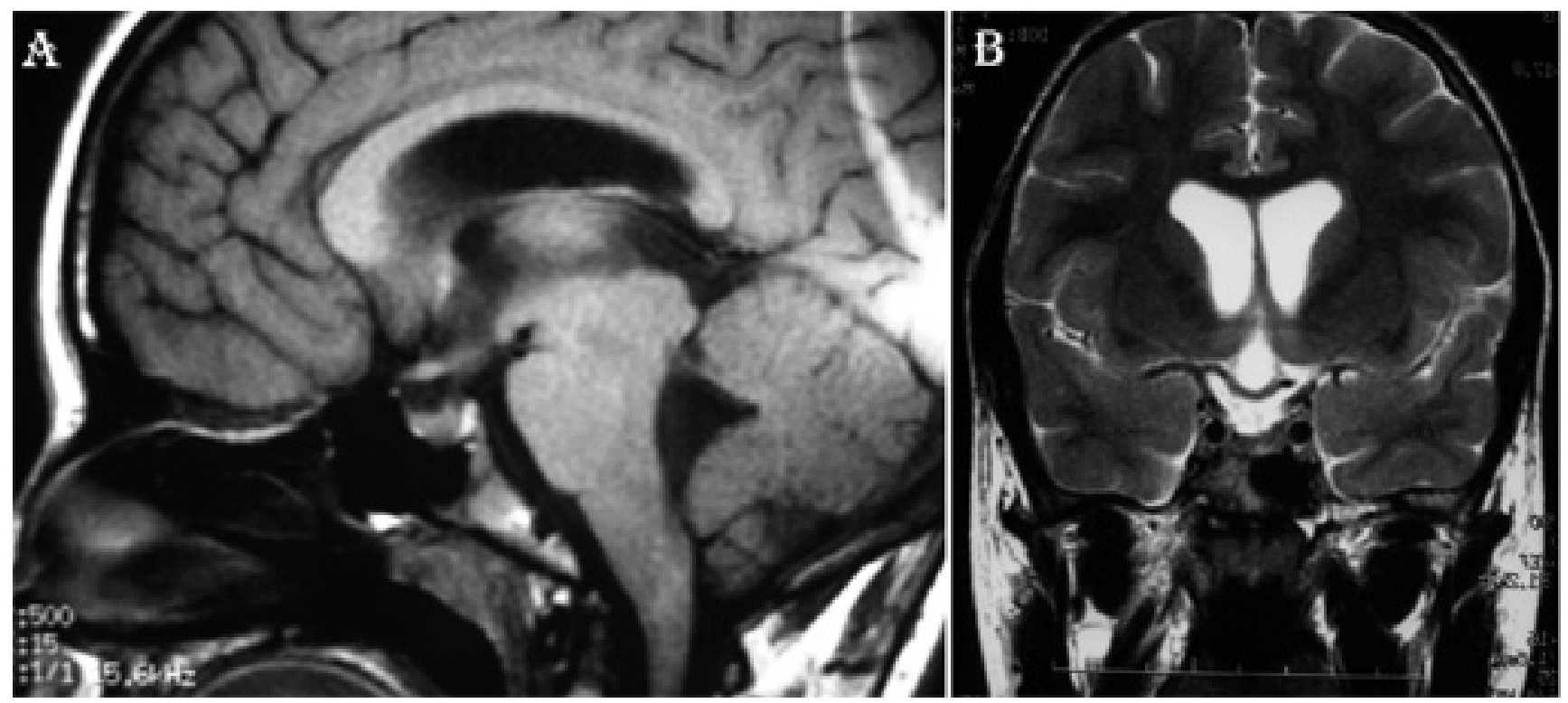

Figura 2. A: TM T1 sagital. B: RM T2 coronal : silla turca vacía parcial durante el episodio de hipertensión intracraneal por malfunción del sistema de derivación de LCR.

monales adenohipofisarios basales no mostraron alteraciones, incluida la alfa-glicoproteína y se decidió, en vista de la ausencia de sintomatología, observar la evolución de la glándula.

En marzo de 2005 ingresó de urgencia por un cuadro de pérdida de visión grave en ambos ojos y papiledema, debido a una rotura del catéter de la derivación ventrículo-peritoneal e hidrocefalia activa. La RM mostró dilatación ventricular y una silla turca vacía parcial (Fig. 2 A y B). Siete horas después de hacerle la RM, se procedió a cambiarle el sistema de derivación de LCR por una válvula programable en presión media, normalizándose la presión intracraneal, aunque quedando como secuela una atrofia óptica con disminución de la agudeza visual. En un control de RM realizado 18 días después, se observó la recuperación del volumen de la glándula a los niveles que tenía antes de la descompensación de la presión intracraneal (Fig. 3 A y B). A los cuatro años de evolución, el volumen de la hipófisis sigue siendo similar, permaneciendo normales las hormonas basales adenohipofisarias y las menstruaciones de la paciente. 

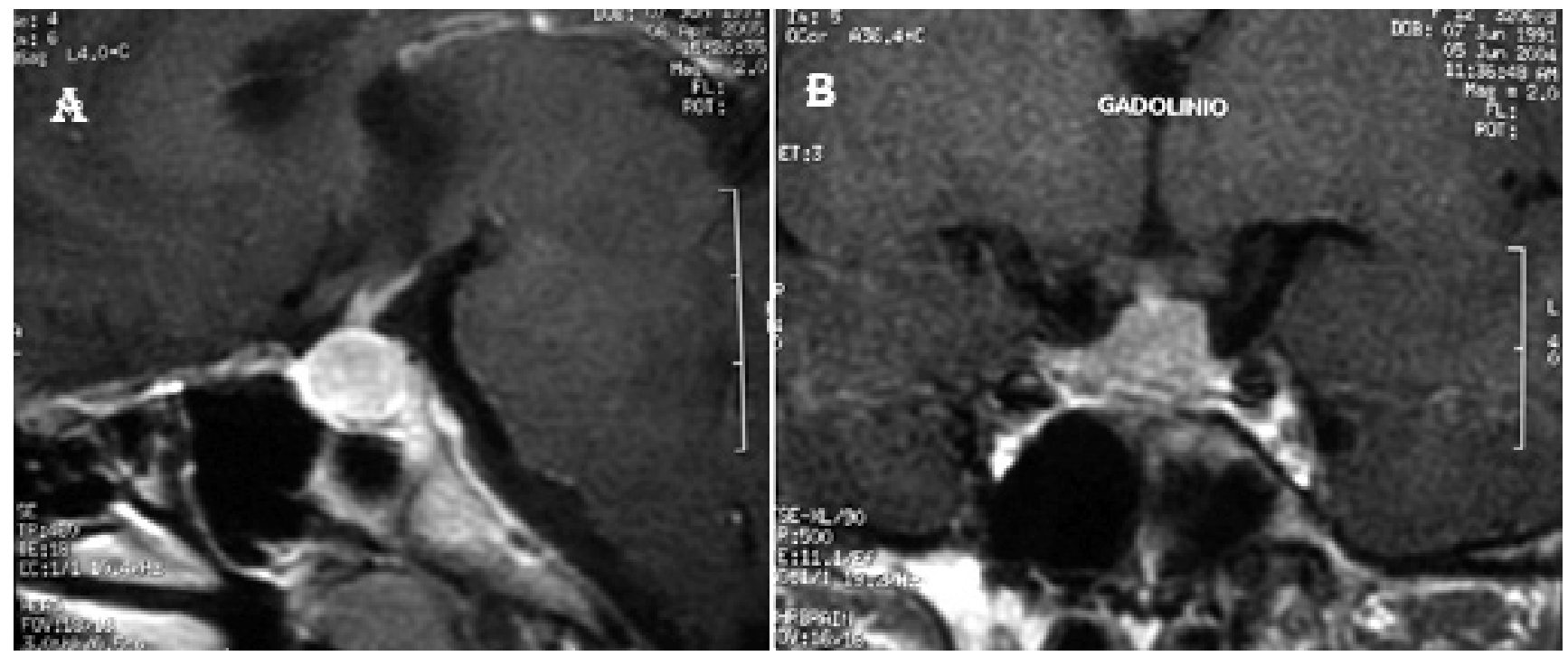

Figura 3. A: RM sagital + contraste. B: RM coronal + contraste 18 días después de la colocación de un nuevo sistema de derivación y normalización de la PIC: La glándula ha recuperado gran parte del volumen que tenía antes del episodio de hipertensión intracraneal.

\section{Comentarios}

La ausencia o incompetencia del diafragma de la silla turca, expone a la glándula hipofisaria a los efectos mantenidos del pulso del LCR, maniobras de Valsalva y de la presión intracraneal. De hecho, la silla turca vacía primaria se asocia a la hipertensión intracraneal crónica idiopática con mucha frecuencia $(10-94 \%)^{4,10,17,19,20}$.

Por otro lado, cuando se realizan estudios de la glándula en la silla turca vacía primaria, se detectan alteraciones hormonales en una proporción no despreciable $(18,7-66,6 \%)^{2,3,5,6,8}$. En general, cuando se hacen estudios funcionales, la incidencia de anomalías supera el 50\% de los casos en la mayoría de las series. Se desconocen los mecanismos por los que se producen estas alteraciones, aunque se ha responsabilizado a la acodadura que sufre el tallo hipofisario sobre el dorso de la silla turca vacía?, que impediría que las hormonas de relación hipotalámicas llegaran hasta las células adenohipofisarias a través de los vasos porta, lo que incluye al factor inhibidor de prolactina (PIF). Maira y col $^{12}$ publicaron 21 pacientes en los que el ritmo y la dinámica de la PRL se normalizaron tras restaurar a valores normales la presión intracraneal, Nosotros tenemos un caso en el que la hiperprolactinemia se normalizó tras colocar una derivación de LCR. Pero la evidencia es escasa.

En contraposición a la incidencia de alteraciones adenohipofisarias, la diabetes insípida es muy rara en la $\operatorname{STVP}(2,8 \%)^{3}$, lo que induce a pensar que existe una distinta susceptibilidad en la hipófisis anterior con respecto a la posterior, a los efectos de la presión intracraneal sobre ellas. Esta distinta vulnerabilidad, puede venir condicionada por la diferencia en la irrigación sanguínea de esas dos partes de la glándula, ya que mientras la neurohipófisis recibe un excelente aporte arterial directo, la adenohipófisis está irrigada casi de manera exclusiva por los vasos porta, de naturaleza venosa ${ }^{14,15,21}$, con presiones sanguíneas intraluminares muy bajas, en el rango de $2,9 \mathrm{~mm}$ de $\mathrm{Hg}^{7}$.

En teoría, cuando no existe un diafragma sellar competente que proteja a la glándula, la presión intracraneal puede condicionar, a la larga, una atrofia isquémica de la adenohipófisis que limita tanto la recuperación de su volumen, como la recuperación funcional tras corregir la hipertensión intracraneal. A favor de esta hipótesis se encuentra el hecho de que las alteraciones hormonales apenas se normalizan tras corregir la hipertensión intracraneal ${ }^{12}$ y el que el volumen de la glándula solo mejora en el 16\% de los casos en la evolución de la STVP 6 .

Sin embargo, esta atrofia grandular puede representar un estadio tardío en la evolución de la silla turca vacía primaria y el caso que presentamos muestra cómo una hipertensión intracraneal aguda puede provocar el desarrollo de una silla turca vacía parcial y su posterior recuperación, cuando la presión intracraneal es normalizada y aún no ha dado tiempo a que se desarrolle la atrofia del parénquima hipofisario. Zagardo y col. ${ }^{22}$ publicaron un caso de HICI en el que la glándula recuperó su volumen normal tras normalizar la presión intracraneal con acetazolamida. La paciente llevaba sólo unas semanas con sintomatología cuando inició el tratamiento, y el volumen de la glándula se normalizó a las dos semanas de tratamiento. También se han publicado dos casos en los que 
la STVP ha revertido tras derivaciones de LCR en HICI; uno de ellos tras derivación ventrículo-peritoneal ${ }^{11} \mathrm{y}$, el otro, tras derivación lumbo-peritoneal ${ }^{22}$. La valoración de estos dos últimos pacientes es más problemática, ya que, en el caso de Lee ${ }^{11}$, la presión de cierre valvular se colocó a propósito en niveles muy bajos $\left(2-5 \mathrm{~cm}\right.$ de $\left.\mathrm{H}_{2} \mathrm{O}\right)$. En el paciente con derivación lumbo-peritoneal, no se comprobó la presión intracraneal tras recuperar la hipófisis su volumen normal, por lo que no es posible descartar el desarrolo de una hipotensión intracraneal dada la inestabilidad de estos sistemas. Es sabido que en los cuadros de hipotensión de LCR, como se ha demostrado mediante estudios de RM, la hipófisis aumenta su tamaño de forma anormal ${ }^{11,13,18}$, lo que se ha atribuido a una hipervolemia pituitaria secundaria al aumento de volumen sanguíneo intracraneal -en acuerdo con la doctrina de Monro-Kellie- que se refleja de manera primaria en la dilatación de los senos venosos y venas meníngeas ${ }^{13,16}$. Lo que sí demuestran estos dos casos es que, en el momento de colocar la derivación de LCR, la glándula pituitaria no estaba atrofiada y su elasticidad le permitió aumentar de nuevo su volumen.

Nuestra paciente presentaba una glándula aumentada de volumen y cuando sufrió una hipertensión intracraneal aguda por rotura del catéter de la derivación, la glándula disminuyó de tamaño, con resultado de una silla turca vacía parcial. Bastó la restauración de la presión intracraneal a sus valores normales, para que la glándula volviera casi a su tamaño original. Esto implica que el diafragma sellar en esta enferma es incompetente y que la glándula conservaba la elasticidad suficiente como para comprimirse y volverse a expandir en razón a los cambios de presión que actuaron sobre ella. En los casos de HICI en los que permanece la silla turca vacía tras restaurar la presión intracraneal, es lógico suponer que la glándula ha perdido la capacidad de recuperar el volumen normal, lo que puede constituir un indicio indirecto de la atrofia isquémica de su parénquima.

\section{Conclusiones}

La silla turca vacía primaria en presencia de hipertensión intracraneal, puede ser un fenómeno reversible si se normaliza la presión antes de que se produzca la involución definitiva de la glándula y, la recuperación de su volumen, es útil como elemento de referencia adicional de la normalización de la PIC.

\section{Bibliografía}

1. Alvarez-Linera, J., Escribano, J., Benito-León, J., PortaEtessam, J., Rovira, A.: Pituitary enlargement in patients with intracranial hypotension syndrome. Neurology. 2000; 55: 1895-1897.

2. Becejac, B., Vizner, B., Berkovié, M., Gnjidié, Z.,
Vrkljan, M.: Neuroendocrinological Aspects of Primary Emty Sella. Coll Antropol 2002; 26 Suppl: 159-164.

3. Biaconcini, G., Bragagni, G., Biaconcine, M.: Primary empty sella syndrome. Observations on 71 cases. Recenti Prog Med 1999; 90: 73-80.

4. Brodsky, M.C., Vaphiades, M.: Magnetic resonante imaging in pseudotumor cerebri. Ophthalmology 1998; 105: 1686-1693.

5. Cannavo, S., Curtó, L., Venturino, M., et al.: Abnormalties of hypothhalacmic.pituitary-thyroid axis in patients with primary empty sella. J Endocrinol Invest 2002; 25: 236239.

6. De Marinis, L., Bonadonna, S., Bianchi, A., Maria, G., Giustina, A.: Extensive clinical experience. Primary Empty Sella. J Clin Endocrinol Metab 2005; 90: 5471-5477.

7. Findell, P.R., Mulchahey, J.J., Shepard, J.M., et al.: Direct measurement of blood pressure within the long hypophysial portal blood vessels. Neuroendocrinology 1987; 45: 263-266.

8. Gasperi, M., Aimaretti, G., Cecconi, E., y col.: Impairment of GH secretion in adults with primary empty sella. J Endocrinol Invest 2002; 25: 329-333.

9. Gharib, H., Harald, M., Laws, E.R., et al.: Coexistent primary empty sella Syndrome and hyperprolactinemia. Arch Intern Med. 1983; 143: 1383-1386.

10. Gibby, W.A., Cohen, M.S., Goldberg, H.I., Sergott, R.C.: Pseudotumor cerebri: CT findings and correlation with vision loss. AJR Am J Roentgenol. 1993; 160: 143-146.

11. Lee, T.C., Yang, L.C., Huang, P.L.: Treatment of empty sella syndrome with ventriculoperitoneal shunt. J Clin Neurosci 2005; 12: 201-205.

12. Maira, G., Anile, C., De Marinis, L., Mancini, A., Barbarino, A.: Cerebrospinal fluid pressure an prolactin in empty sella syndrome. Can J Neurol Sci 1990; 17: 92-94.

13. Mokri, B.: Intracranial hypertension after treatment of spontaneous cerebrospinal fluid leaks. Mayo Clin Proc 2002; 77: 1241-1246.

14. Monnet, F., Elias, K.A., Fagin, K., Neill, A., Goldsmith, P., Weiner, R.I.: Formation of a direct arterial blood supply to the anterior pituitary gland following complete or partial interruption of the hypophyseal portal vessels. Neuroendocrinology 1984; 39: 251-255.

15. Page, R.B., Bergland, R.M.: Pituitary vasculature. En Allen M.B. Jr., Mahesh V.B., (ed): The Pituitary: a Current Review. New York; Academic Press, 1977, pp 9-17.

16. Settipani, N., Piccoli, T., La Bella, V., Piccoli, F.: Cerebral venous sinus expansion in post-lumbar puncture headache. Funct Neurol 2004; 19: 51-52.

17. Silbergleit, R., Junck, L., Gebarski, S.S., Hatfield, M.K.: Idiopathic intracranial hypertension (pseudotumor cerebri): MR imaging. Radiology 1989; 170: 207-209.

18. Shimazu, N., Oba, H., Aoki, S., Monzawa, S., Makita, K., Araki, T.: Pituitary enlargement in spontaneous intracraneal 
hypotension on MRI. Nippon Igaku Hoshasen Gakkai Zasshi. 1998; 58: 349-352.

19. Weisberg, L.A.: Computed tomography in benign intracranial hypertension. Neurology 1985; 35: 1075-1078.

20. Wessel, K., Thron, A., Linden, D., Petersen, D., Dichgans, J.: Pseudotumor cerebri: clinical and neuroradiological findings. Eur Arch Psychiatry Neurol Sci 1987; 237: 54-60.

21. Xuereb, G.P., Prichard, M.M., Daniel, P.M.: The arterial supply and venous drainage of the human hypophysis cerebri. Q J Exp Physiol Cogn Med Sci 1954; 39: 199-217.
22. Zagardo, M.T., Cail, W.S., Kelman, S.E., Rothman, M.I.: Reversible empty sella un idiopathic intracraneal hipertensión: an indicador of successful therapy? AJNR 1996; 17: 1953-1956.

González-Tortosa, J.; Piqueras-Pérez, C.; Ruiz-Espejo, A.; Martínez-Lage, J.F.: Silla turca vacía primaria reversible. A propósito de un caso. Neurocirugía 2010; 21: 317-321.

Correspondencia: J. González-Tortosa. Servicio de Neurocirugía. Hospital Universitario Virgen de la Arrixaca. Murcia. 